\title{
Erratum to: Late diagnosis of a McFarland fracture: imaging and treatment
}

Panagiotis D. Symeonidis • George Ath Konstantinidis • Panagiotis S. Dionellis • John Ousantzopoulos • Panagiotis K. Givissis

Published online: 2 August 2013

(C) ISS 2013

Erratum to: Skeletal Radiol

DOI 10.1007/s00256-013-1680-x

The original version of this article unfortunately contained spelling errors. The names of "John Ousantzopoulos and Panagiotis K. Givissis" are now corrected in the author group of this article.

The online version of the original article can be found at http:// dx.doi.org/10.1007/s00256-013-1680-x.

P. D. Symeonidis $\cdot$ G. A. Konstantinidis $(\triangle) \cdot$ P. K. Givissis Aristotelian University of Thessaloniki, E. Aminis 18, Thessaloniki, Greece 54621

e-mail: konstantigeo@gmail.com

P. S. Dionellis

Hippocration General Hospital of Thessaloniki, Konstantinoupoleos

49 Street, 54642 Thessaloniki, Greece

J. Ousantzopoulos

Private Practice Thessaloniki, Iviskou 7, 54531 Thessaloniki, Greece

G. A. Konstantinidis

Aristotelian University of Thessaloniki, University Campus

Thessaloniki, 54124 Thessaloniki, Greece

P. K. Givissis

Aristotelian University of Thessaloniki, Eksoxi 57010, Thessaloniki,

Greece 